\title{
Diesel Engine Emission Reduction Using Catalytic Nanoparticles: An Experimental Investigation
}

\author{
Ajin C. Sajeevan and V. Sajith \\ School of Nano Science and Technology, National Institute of Technology, Calicut 673 601, India \\ Correspondence should be addressed to V. Sajith; sajith@nitc.ac.in
}

Received 6 November 2012; Revised 25 January 2013; Accepted 29 January 2013

Academic Editor: Dmitry Murzin

Copyright ( 2013 A. C. Sajeevan and V. Sajith. This is an open access article distributed under the Creative Commons Attribution License, which permits unrestricted use, distribution, and reproduction in any medium, provided the original work is properly cited.

Cerium oxide being a rare earth metal with dual valance state existence has exceptional catalytic activity due to its oxygen buffering capability, especially in the nanosized form. Hence when used as an additive in the diesel fuel it leads to simultaneous reduction and oxidation of nitrogen dioxide and hydrocarbon emissions, respectively, from diesel engine. The present work investigates the effect of cerium oxide nanoparticles on performance and emissions of diesel engine. Cerium oxide nanoparticles were synthesized by chemical method and techniques such as TEM, EDS, and XRD have been used for the characterization. Cerium oxide was mixed in diesel by means of standard ultrasonic shaker to obtain stable suspension, in a two-step process. The influence of nanoparticles on various physicochemical properties of diesel fuel has also been investigated through extensive experimentation by means of ASTM standard testing methods. Load test was done in the diesel engine to investigate the effect of nanoparticles on the efficiency and the emissions from the engine. Comparisons of fuel properties with and without additives are also presented.

\section{Introduction and Background}

Diesel engines are blessed with high thermal efficiency and hence widely used in automobiles. However diesel engines are one of the major contributors to the emissions such as hydrocarbons, particulates, nitrogen oxides, and sulphur oxides. These emissions are very harmful to human beings and also responsible for acid rain and photochemical contamination and hence subject to strict environmental legislation. Thus wide use of diesel engines leads to harmful threat of nitrogen oxide and hydrocarbon emissions. Improvement in the performance of diesel engines is an important challenge to be addressed, in the current era due to the fast depletion of fossil fuel resources as well as due to the harmful hydrocarbon and nitrogen oxide emissions. Efforts are also made for the reformulation of diesel fuel to reduce these harmful emissions without affecting the physicochemical properties of fuel such as viscosity, flash and fire point, and so forth [1-3].

Various works have been reported on modified fuels for improving diesel engine performance and reducing exhaust emissions. Kim and Choi [4] carried out experiments with pure diesel, diesel blended with $15 \%$ ethanol, and a cetane number improver EHN (ethyl hexane nitrate). The mean conversion efficiency of $\mathrm{HC}$ and $\mathrm{CO}$ emissions by the catalyst was observed to be $60 \%$ and the PM emission was reduced by $30-40 \%$, while analyzing diesel blended with $15 \%$ ethanol and EHN.

De Menezes et al. [5] experimentally observed that the presence of ethanol and ethyl terbutyl-ether (ETBE) significantly alters the volatility and reduces cetane number. As an oxygenated additive, dimethyl carbonate (DMC) is usually blended with diesel fuel to improve combustion and reduce emissions of diesel engines. Experiments by Xiaolu et al. [6] show that the dimethyl carbonate fueled engine has lower nitrogen oxides emissions and 2-3\% higher effective thermal efficiency as compared to the engines operated with diesel in moderate and high load zones.

Experimental results have been reported on the influence of various fuel additives such as organic compounds of manganese, magnesium, copper, and calcium on fuel properties. It was observed that organic compounds of copper, magnesium, and calcium are less effective in comparison with that of manganese [3]. Shi et al. [7] and Satgé de Caro et al. [8] reported that the particulate matter emissions decrease with 
increasing oxygenate content in the fuels. The effect of methanol-containing additive (MCA) on the emission of carbonyl compounds, generated from the diesel engine, was studied by Chao et al. [9] and high emissions were observed with the use of MCA.

Metal oxides such as those of copper, iron, cerium, and cobalt have been used as fuel additives. Among these oxides, cerium oxide [10] is the most abundant element in rare earth family with good thermal stability as well as cross-over efficiency which will undergo redox cycling between the trivalent and tetravalent oxidation states. Cerium oxide, when used in the nanoparticles form exhibits a high catalytic activity because of its high surface-to-volume ratio leading to improvement in the fuel efficiency and reduction in the emissions. Sajith et al. [11,12] and Selvan et al. [13] investigated the effect of cerium oxide nanoparticles on the engine performance and the emissions, with diesel and bio-diesel as fuel. The brake thermal efficiency of the engine was found to be increased by $5 \%$ and a drastic reduction in both $\mathrm{HC}$ and $\mathrm{NO}_{x}$ emissions was observed.

Various methods for the synthesis of metal, metal oxide, and nonmetallic nanoparticles include chemical, physical, physicochemical top down and bottom up methods. Chen and Chang $[14,15]$ explained the synthesis of cerium oxide nanoparticles by homogenous precipitation method with mixtures of alcohol and water as solvents. Six alcohols such as methanol, ethanol, n-propanol, iso-propanol, tertabutanol, and ethylene glycol (EG) were separately mixed with water as the solvent. It was reported that the synthesized nanoparticles with cubic fluorite structure have a size range of 7-15 $\mathrm{nm}$ and the least diameter was obtained with ethylene glycol as the solvent. As the percentage volume of alcohol increases, the particle size decreases for all solvent systems. Ivanov et al. [16] synthesized nanocrystalline cerium oxide with cerium nitrate hexahydrate salt as precursor. The solvent used was waterisopropanol mixture and their ratio was the only varying parameter which directly affects the crystallization and size of nanoparticles.

$\mathrm{Gu}$ and Soucek [17] explained the preparation and characterization of uniform sized monodispersed cerium oxide nanoparticles in hydrocarbon solvents in one-step reaction, by heating cerium oleate complex in an organic solvent with high boiling point, to decompose and form nanocrystals. The size of the nanoparticles depends on type of solvent, reaction time, reactant concentration, and so forth. Masui et al. [18] reported the microemulsion method or reverse micelle method for synthesis of high activity catalytic cerium oxide nanoparticles. The microemulsion method basically consists of a reaction within reversed micelle in a thermodynamically stable system composed of two immiscible liquids and a surfactant. Aruna and Mukasyan [19] proposed an effective low cost method for the production of wide range of fine sized nanoparticles by combustion synthesis or self-propagating high-temperature synthesis (SHS). Mädler et al. [20] explained flame spray pyrolysis (FSP) which is a promising technique for synthesis of high purity nanosized materials with controlled size and crystallinity in one step. In this method a flame is used to force chemical reactions of precursor compounds, finally resulting in the formation of clusters, which increase their size to a range of some nanometres by coagulation and sintering.

The effect of cerium oxide on the size distribution and composition of diesel particulate matter has been studied by Skillas et al. [21], which indicated a reduction in the accumulation mode, but an increase in ultrafines. The catalytic effect of cerium oxide on diesel particulate matter oxidation has been studied by Lahaye et al. [22] and observed that cerium oxide is present both on and within the soot as cerium oxides. Neeft et al. [23] identified the metal oxides of copper and cerium as additives which will cause spontaneous regeneration as they uniformly distribute the particulates or soot, by catalyzing the combustion during regeneration. Stanmore et al. [24] performed experiments on the ignition and combustion of cerium oxide doped diesel soot and the results show a significant increase in intrinsic oxidation rate with the presence of the metal additive.

Jung et al. [25] carried out investigation on the influence of cerium oxide additive on the kinetics of oxidation and size distribution of ultrafine diesel particles using a hightemperature oxidation-tandem differential mobility analysis method over the temperature range $300-700^{\circ} \mathrm{C}$. The addition of cerium in diesel fuel was observed to cause significant changes in number weighted size distributions, light-off temperature, and kinetics of oxidation. The light-off temperature was reduced by 250 and $300^{\circ} \mathrm{C}$, respectively, for 25 and $100 \mathrm{ppm}$ dosing levels. The oxidation rate was observed to be increased significantly with the addition of cerium oxide in the fuel even though the rate was relatively insensitive to dosing level. The addition of cerium oxide likely increased the number of catalytic sites but had less effect on the overall activation energy due to the presence of other metals in the diesel particulate matter coming from lubricating oil.

The present experimental study aims at the investigation of the effect of cerium oxide $\left(\mathrm{CeO}_{2}\right)$ in the form of nanoparticles, as an additive in diesel, on the physicochemical properties of diesel as well as the engine performance and emissions. The work also involves the synthesis of cerium oxide nanoparticles by precipitation method which is a chemical method. The fuel properties tested include flash and fire points and viscosity [26, 27]. Single cylinder water-cooled direct injection diesel engine was used for conducting the performance tests. Engine performance and emission characteristics were obtained and analyzed. The experimental investigations were carried out by varying the dosing levels of $\mathrm{CeO}_{2}$ nanoparticles in the fuel (from 5 to $35 \mathrm{ppm}$ ). The study of the stability of the nanofluid also has been carried out, with the addition of surfactants in the diesel, in the present work.

\section{Experimental Study}

The experimental investigations were carried out in three phases. In the first phase, cerium oxide nanoparticles were prepared by chemical method and added to diesel to obtain a stable suspension. In the second phase various physicochemical properties of modified diesel were determined and compared with those of the base fuel. Standard ASTM test procedures were adopted to determine different properties 
such as flash and fire points and viscosity. In the third phase, performance tests were conducted on a single cylinder diesel engine using the modified and base fuels, in order to evaluate the engine performance as well as the emission characteristics, using an exhaust gas emission analyzer. The method of synthesis of cerium oxide nanoparticles, preparation of the fuels with the additive nanoparticles, along with the experimental methods for obtaining the fuel properties and the details of the performance test facility are discussed below.

2.1. Synthesis of Cerium Oxide Nanoparticles. Cerium oxide nanoparticles were synthesized by means of precipitation method. Chemicals used in this method were reagent-grade cerium nitrate $\mathrm{Ce}\left(\mathrm{NO}_{3}\right)_{3} \cdot 6 \mathrm{H}_{2} \mathrm{O}$ (Sigma-Aldrich, purity 99\%), analytical-grade iso propanol, and aqueous ammonia. The reaction was carried out at room temperature. $0.08 \mathrm{M}$ cerium (III) nitrate solution in water-iso propanol mixture in the volume ratio of 1:6 was prepared and was vigorously stirred by means of a magnetic stirrer. A fivefold excess of $3 \mathrm{M}$ aqueous ammonia solution was added to the solution. $\mathrm{pH}$ of the solution was adjusted to 10 by the addition of ammonium hydroxide, as alkaline medium gives smaller particles than acidic. After one hour the pale red colored reactants turns to yellow, indicating the formation of cerium oxide nanoparticles. The following equations show the series of chemical reactions occurring during formation of cerium oxide, in this method.

Precipitation of Cerium hydroxide:

$$
\begin{array}{r}
8 \mathrm{Ce}\left(\mathrm{NO}_{3}\right)_{3}+22 \mathrm{NH}_{4} \mathrm{OH}+7 \mathrm{H}_{2} \mathrm{O} \\
\longrightarrow 8 \mathrm{Ce}(\mathrm{OH})_{4}+23 \mathrm{NH}_{4} \mathrm{NO}_{3}
\end{array}
$$

Conversion of amorphous cerium hydroxide to cerium oxide on heating:

$$
\mathrm{Ce}(\mathrm{OH})_{4}+\mathrm{Heat} \longrightarrow \mathrm{CeO}_{2}+2 \mathrm{H}_{2} \mathrm{O}
$$

These synthesized nanoparticles were calcinated at $500^{\circ} \mathrm{C}$ for 3 hours for obtaining crystalline nanosized particles. A surfactant has been used for obtaining better dispersion of ceria nanoparticles in diesel. The selection of surfactant depends on the hydrophilic lipophilic balance (HLB) of the surfactant, which is the relative degree to which the surfactant is water soluble or oil soluble. In the present work the surfactant used was dodecenyl succinic anhydride (DDSA$\mathrm{C}_{16} \mathrm{H}_{28} \mathrm{O}_{3}$ ), which has a HLB value of 1.34 and is a stable compound with adhesive property, slight odour and is totally soluble in diesel. The lipophobic part of the DDSA molecule is embedded into the cerium oxide particle, leaving the lipophilic part of the molecule to interact with diesel. Addition of DDSA in diesel will also improve the detergent properties.

Two samples of cerium oxide, one with surfactant coating and the other without coating were synthesized and characterized by means of TEM, EDS, and XRD. The elemental composition of the synthesized ceria particles was obtained by means of energy dispersive spectroscopy as shown in Figure 1. The weight percentage and atomic percentage of cerium
TABLE 1: EDS analysis of cerium oxide nanoparticles.

\begin{tabular}{lcc}
\hline Element & Weight \% & Atomic \% \\
\hline $\mathrm{O}(\mathrm{K})$ & 17.43 & 64.9 \\
$\mathrm{Ce}(\mathrm{L})$ & 82.57 & 35.10 \\
\hline Total & 100 & 100 \\
\hline
\end{tabular}

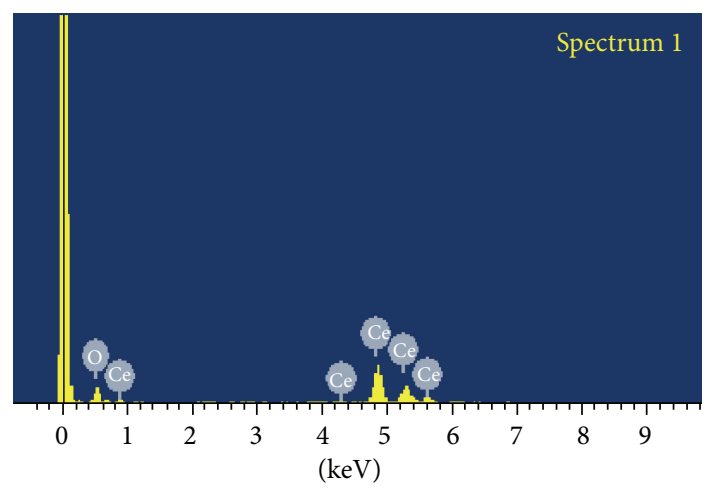

Full scale 2030 cts cursor: 0

Figure 1: EDS spectrum of $\mathrm{CeO}_{2}$ nanoparticles.

present in the synthesized sample are quantitatively represented in Table 1. Figures 2(a) and 2(b) show the TEM images of cerium oxide nanoparticles, with and without surfactant coating, respectively. A size range $40-50 \mathrm{~nm}$ was observed from TEM images and the size was found to be reduced with the addition of surfactant DDSA, as it helps to reduce the chance of aggregation of nanoparticles. The cubic fluorite structure of cerium oxide and crystallite size was confirmed from XRD (Bruker AXS D8) data and Figure 3 shows the X-Ray Diffraction pattern. XRD pattern shows three main reflections (111), (220), and (200) as characteristic of $\mathrm{CeO}_{2}$ cubic phase with fluorite structure. The solid solution of synthesized nanoparticles shows peaks similar to $\mathrm{CeO}_{2}$ nanoparticles. It is clear from the powder XRD data that a phase pure crystalline cerium zirconium is formed. The peaks became broader on addition of DDSA which is an indication of reduction in particle size.

2.2. Preparation of Nanoparticle Added Fuel. The dosing level of cerium oxide nanoparticle samples (by weight) in diesel was varied from 5 to $40 \mathrm{ppm}$. In order to obtain a uniform suspension of nanoparticles in diesel, a standard ultrasonic shaker (Power Sonics 405) has been used for mixing the nanoparticles corresponding to the required dosing level. The catalytic nanoparticle added diesel was agitated for about 30 minutes in an ultrasonicator to obtain a stable nanofluid. The modified fuel was used in the experiments immediately after preparation, so that considerable time is not allowed for sedimentation to set in.

2.3. Stability Studies. As stability of nanofluid is of much concern, stability studies of nanoparticle added diesel have been carried out with the help of Dynamic Light Scattering system (Zetasizer Nano-ZS, Malvern). Nanoparticles in 


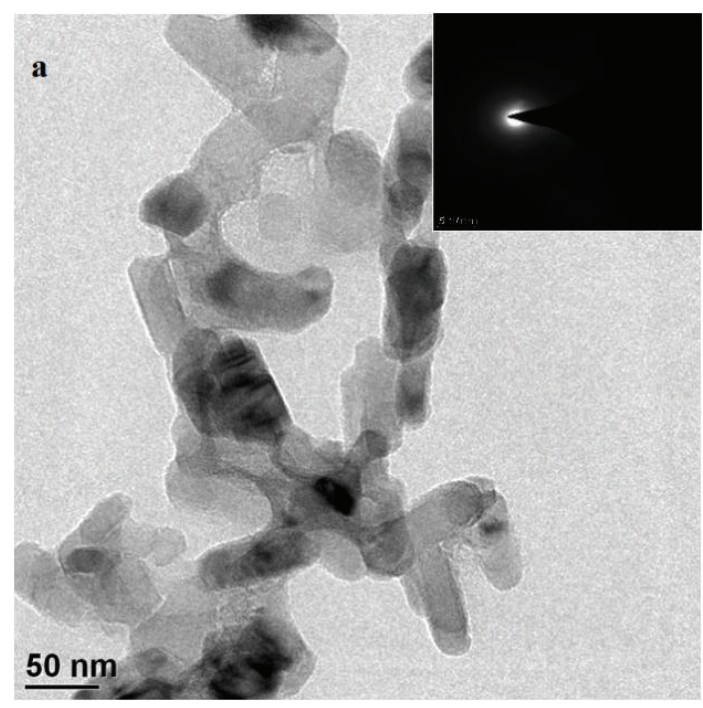

(a)

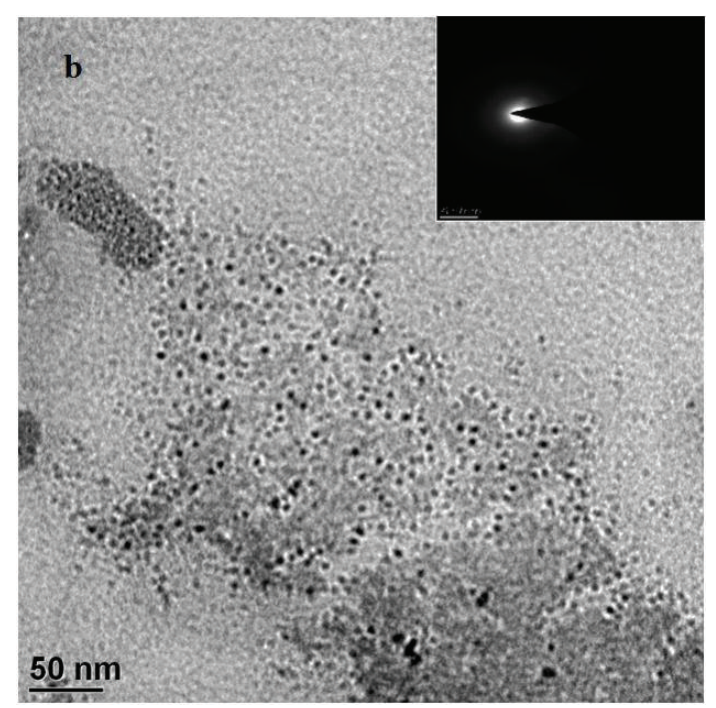

(b)

FIGURE 2: TEM images of cerium oxide nanoparticles (a) without DDSA and (b) with DDSA.

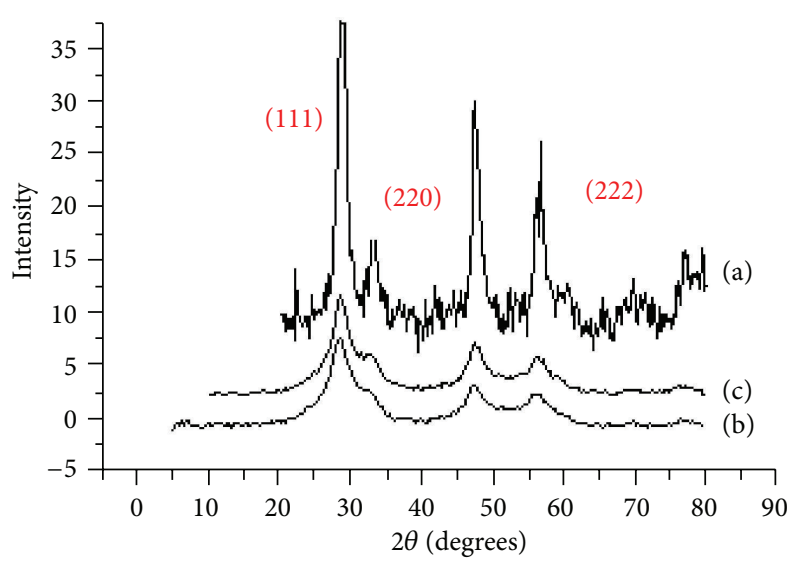

(a) Cerium oxide

(b) Cerium oxide with $2 \%$ DDSA

(c) Cerium oxide with $5 \%$ DDSA

FIGURE 3: X-Ray Diffraction pattern of cerium oxide nanoparticles.

a dispersion medium always undergo brownian motion and the stability of nanofluids is thus determined by the interaction between the particles during their collision. Among the two basic interactions, that is, attractive and repulsive, when repulsion dominates, the system will be stable and will remain in a dispersed state. Zeta potential which is the potential difference between the dispersion medium and the stationary layer of fluid attached to the dispersed particle is a measure of the stability of nanofluids. Zeta potential of nanofluids can be improved by the addition of surfactant and a higher zeta potential indicates a better stable dispersion. The zeta potential of the modified fuels was measured using dynamic light scattering technique and is shown in Table 2. Table 2 shows
TABLE 2: Zeta potential values of modified diesel fuel.

\begin{tabular}{llc}
\hline Sl no. & Sample & Zeta potential \\
\hline (1) & 5 ppm Cerium oxide added diesel & $3.80 \mathrm{mV}$ \\
(2) & 15 ppm Cerium oxide added diesel & $14.50 \mathrm{mV}$ \\
$(3)$ & 25 ppm Cerium oxide added diesel & $34.50 \mathrm{mV}$ \\
(4) & 35 ppm Cerium oxide added diesel & $37.6 \mathrm{mV}$ \\
& 35 ppm Cerium oxide added diesel with & $46.50 \mathrm{mV}$ \\
(5) & 2\% DDSA & \\
(6) & 35 ppm Cerium oxide added diesel with & $19.5 \mathrm{mV}$ \\
\hline
\end{tabular}

that maximum zeta potential is obtained for the sample containing $35 \mathrm{ppm}$ of cerium oxide nanoparticles, with $2 \%$ volume percentage of DDSA and hence from the stability point of view this sample is the most stable.

2.4. Determination of Fuel Properties. American standard test methods have been employed for the determination of various properties of fuel such as viscosity, flash, and fire points. The viscosity was measured using the redwood viscometer and a cleveland open cup flash and fire point apparatus [24] were used for measuring the flash and fire point [25].

2.5. Engine Performance and Emission Testing. Performance and emission studies have been conducted in a four-stroke, single cylinder, water-cooled compression ignition engine, specifications of which are given in Table 3 . The schematic block diagram of the experimental setup is shown in Figure 4. Standard constant speed load tests were conducted on the engine. A brake drum dynamometer was used to load the engine. 
TABLE 3: Engine specifications.

\begin{tabular}{lc}
\hline Type & $\begin{array}{c}\text { Naturally aspirated, four stroke single cylinder, } \\
\text { water-cooled compression ignition engine }\end{array}$ \\
\hline Stroke & $110 \mathrm{~mm}$ \\
Bore & $88 \mathrm{~mm}$ \\
Rated output & $5.5 \mathrm{Kw}$ \\
Rated speed & $1500 \mathrm{RPM}$ \\
Loading device & Brake drum dynamometer \\
\hline
\end{tabular}

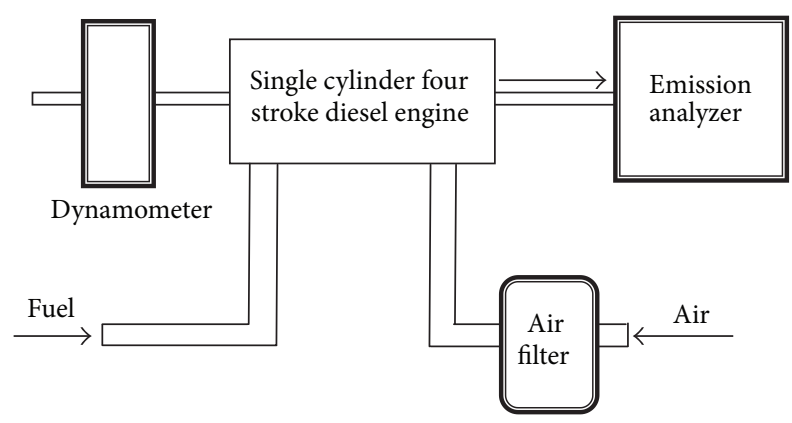

FIGURE 4: Schematic of the experimental setup.

\section{Results and Discussions}

Nanoparticles synthesized in controlled laboratory conditions, by means of precipitation method, were used for the preparation of diesel nanofluids. Dodecyl succinic anhydride, a surfactant, was added for improving the stability of nanofluids. Various physicochemical properties of fuels were determined by standard ASTM test methods. The tests were conducted with diesel and nanoparticle added diesel and the results obtained were compared. Diesel engine performance and exhaust emissions tests were conducted with diesel and nanoparticle added diesel fuel and results were compared.

3.1. Fuel Properties. Various physicochemical properties of both the base fluid (diesel) and nanoparticle added modified diesel were tested by means of standard ASTM methods under identical laboratory conditions. The results obtained were compared in order to investigate the effect of nanoparticle and its dosing level on the fuel properties.

3.1.1. Flash Point. Flash point is the temperature at which a flash is momentarily produced on the introduction of a spark on the surface of a heated fuel and is also an indication of the volatility a fuel. Higher the volatility of a fuel, lower will be the flash point and vice versa. Flash point was tested for the nanoparticle added diesel up to a dosing level of $80 \mathrm{ppm}$. Variation of flash point as a function of concentration of nanoparticles is given in Figure 5, which indicates a decrease in the volatility of the fuel with the nanoparticle addition. Higher flash point temperatures are desirable for safer handling of fuel. As the addition of catalytic nanoparticles in the fuel increases its flash point, nanoparticle added fuel is inherently safer to handle as compared to its base fuel.

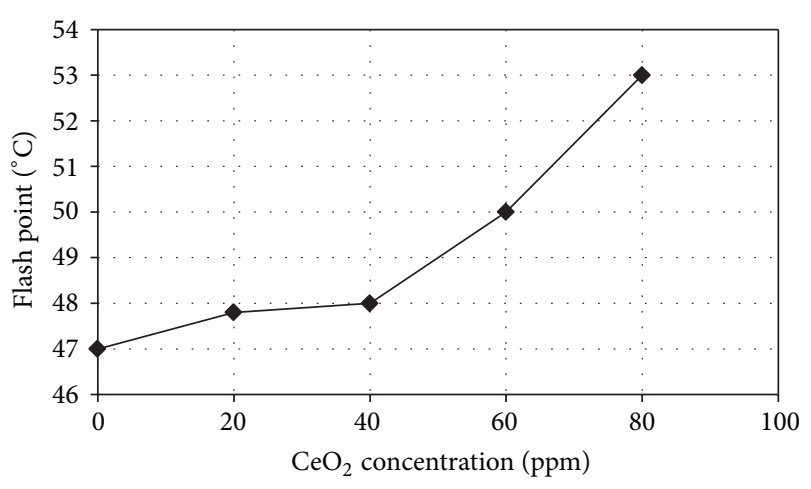

FIGURE 5: Variation of flash point of diesel as a function of concentration of cerium oxide nanoparticles.

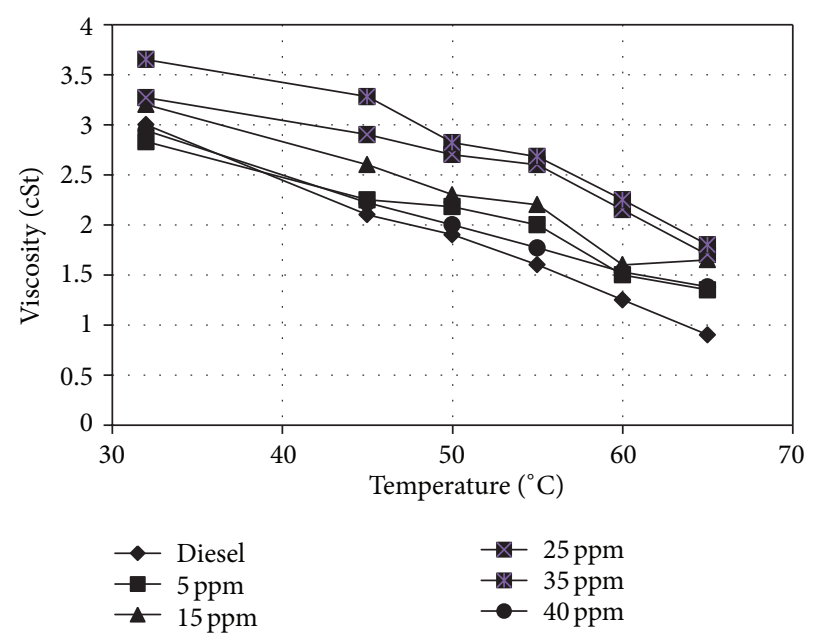

FIgURE 6: Variation of kinematic viscosity of diesel as a function of concentration of cerium oxide nanoparticles.

3.1.2. Kinematic Viscosity. Kinematic viscosity of the diesel is found to increase with nanoparticle addition as shown in Figure 6 . The addition of nanoparticles in the fuel increases the resistance between the fluid layers and hence increases the viscosity. Lower fuel viscosities may not provide sufficient lubrication of fuel injection pumps or injector plungers resulting in increased wear. At the same time, increase in viscosity will adversely affect the atomization leading to an increase in the exhaust emissions. The surfactant DDSA was added to obtain a stable suspension of nanoparticle added diesel. Figure 7 shows the comparison of the viscosity of the diesel with that of nanoparticle added diesel and surfactant treated nanoparticle added diesel at different temperatures. Even though an increase in viscosity was observed on the addition of nanoparticles to the diesel, it was found that the viscosity of diesel decreases on the addition of DDSA to the catalytic nanoparticles. The addition of surfactant in the fuel along with the catalytic nanoparticles not only improves the stability of the nanoparticle fuel suspension, but also reduces the viscosity of diesel. The viscosity of the nanoparticle added diesel, nanoparticle added diesel with surfactant, and pure 


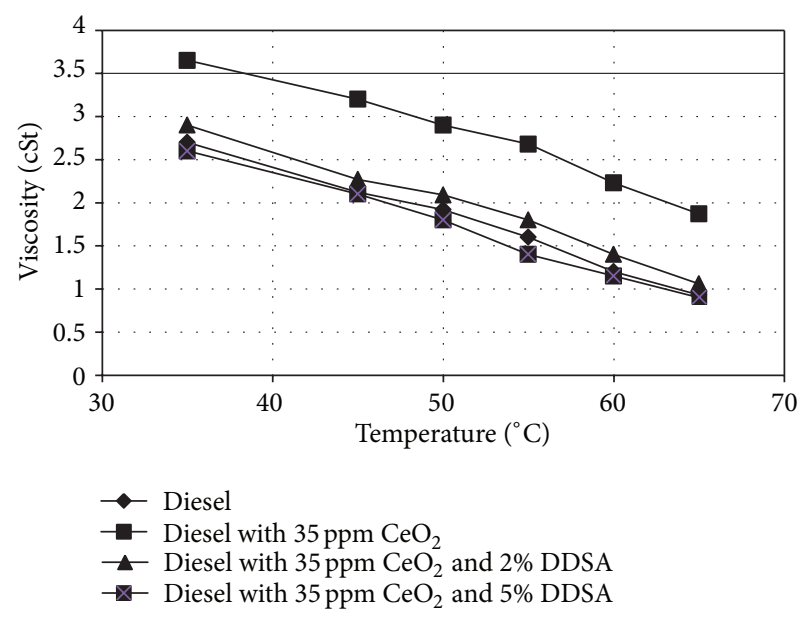

FIGURE 7: Variation of kinematic viscosity of diesel as a function of temperature.

diesel is found to be decreased with the increase in temperature, as expected.

3.2. Engine Performance. The performance tests were conducted on a diesel engine with diesel and modified diesel. Figure 8 shows the variation of brake thermal efficiency with increasing load for different concentrations of cerium oxide nanoparticles. The brake thermal efficiency was increased by about $6 \%$ on the addition of cerium oxide nanoparticle in diesel. The cerium oxide nanoparticles present in the fuel promote longer and more complete combustion as compared to the base fuel, as cerium oxide acts as an oxygen buffer, releasing and storing oxygen depending upon the partial pressure of oxygen. The efficiency of cerium oxide as a catalyst is related to its ability to undergo a transformation from the stoichiometric $\mathrm{CeO}_{2}(+4)$ state to the $\mathrm{Ce}_{2} \mathrm{O}_{3}(+3)$ valence state via a relatively (in comparison with other oxides) low energy reactions. As the dosing level of cerium oxide increases beyond $35 \mathrm{ppm}$ a slight decrease in the efficiency was observed, especially at higher loads, which points to the optimum level of dosing of catalytic nanoparticles in diesel. As the dosing level of nanoparticles is increased, chance of agglomeration will be more which increases the size of nanoparticles, which will affect its catalytic action. The effect of surfactant (DDSA) coated catalytic ceria nanoparticles on performance of diesel engine performance has also been investigated. Figure 9 shows the variation of brake thermal efficiency for diesel and diesel with a dosing level of $35 \mathrm{ppm}$ of catalytic nanoparticles along with the surfactant DDSA of different concentrations. The efficiency was found to be increased with the addition of DDSA especially at higher loads, even though the effect of DDSA is not pronounced at lower loads. The addition of DDSA in the fuel decreases the viscosity leading to better atomization of fuel droplets and hence improvement in the efficiency. A drop in brake thermal efficiency was also observed when the dosing level of DDSA was increased from 2 to $5 \%$, putting a limit to the dosing of surfactant in the fuel.

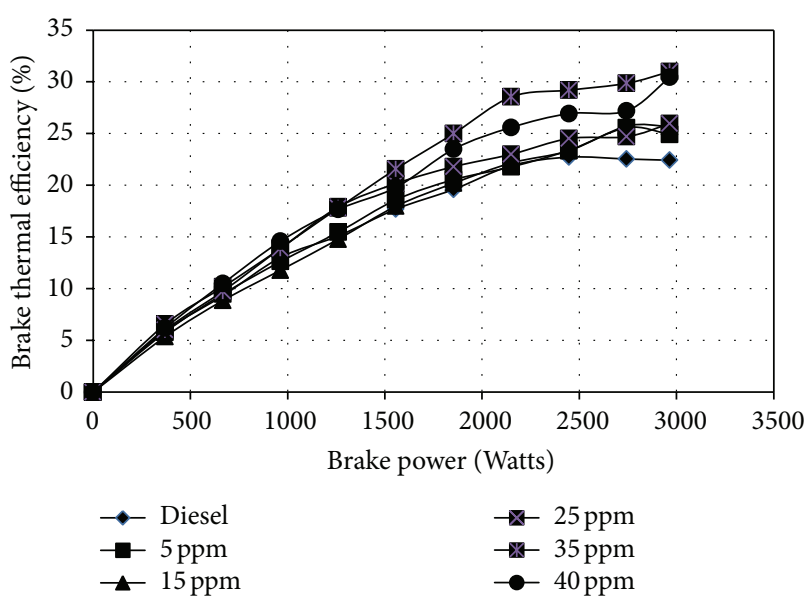

FIGURE 8: Variation of the brake thermal efficiency with load.

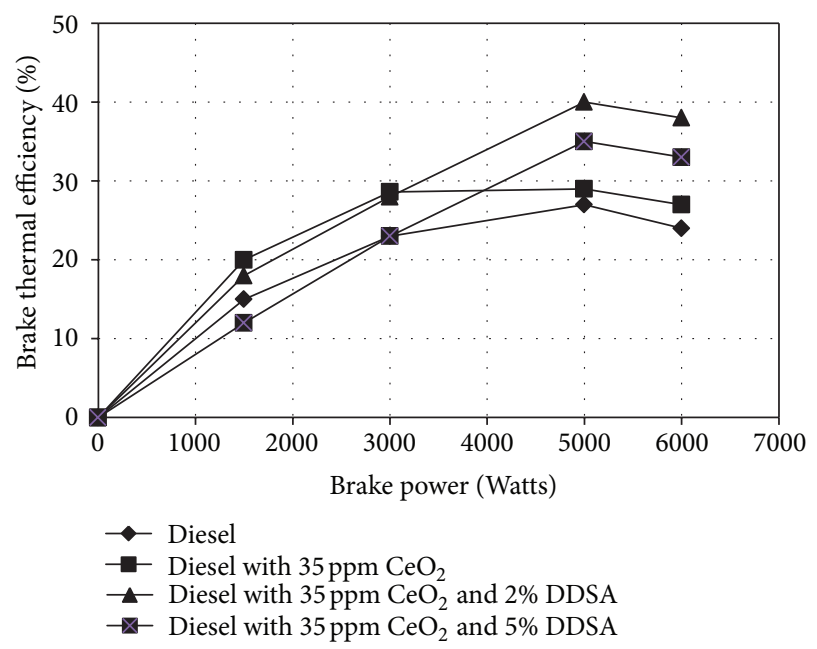

FIGURE 9: Variation of the brake thermal efficiency with load, for diesel and modified diesel ( $35 \mathrm{ppm}$ of $\mathrm{CeO}_{2}$ ) with different vol \% of surfactant.

3.3. Exhaust Emissions. The load tests were conducted by varying the dosing level of cerium oxide nanoparticles in the diesel and the emissions such as hydrocarbon and nitrogen oxide emissions were measured using a standard emission analyzer (KM9106 Quintox Combustion Analyser).

3.3.1. Hydrocarbon Emissions. The hydrocarbon emissions were decreased on addition of catalytic nanoparticles by about 40 to $45 \%$, especially at higher load as shown in Figure 10. It was also observed that the reduction in the HC emissions increases with the increase in the concentration of cerium oxide from $5 \mathrm{ppm}$ to $35 \mathrm{ppm}$. Cerium oxide has the ability to undergo a transformation from the stoichiometric $\mathrm{CeO}_{2}(+4)$ valance state to the $\mathrm{Ce}_{2} \mathrm{O}_{3}(+3)$ state, releasing the oxygen. Thus cerium oxide supplies the oxygen for the oxidation of the hydrocarbon as well as the soot and gets converted to cerous oxide $\left(\mathrm{Ce}_{2} \mathrm{O}_{3}\right)$ as follows [28]. 


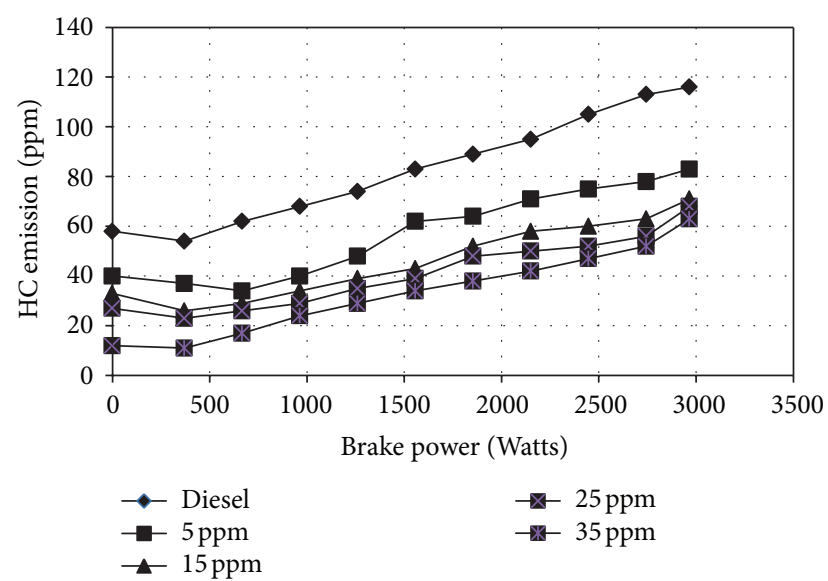

FIGURE 10: Variation of HC emissions with load for diesel and modified diesel.

Hydrocarbon combustión:

$$
\begin{aligned}
& (2 x+y) \mathrm{CeO}_{2}+\mathrm{C} x \mathrm{H} y \\
& \quad \longrightarrow\left[\frac{(2 x+y)}{2}\right] \mathrm{Ce}_{2} \mathrm{O}_{3}+x \mathrm{CO}+\frac{y}{2} \mathrm{H}_{2} \mathrm{O}
\end{aligned}
$$

Soot burning:

$$
4 \mathrm{CeO}_{2}+\text { Csoot } \longrightarrow 2 \mathrm{Ce}_{2} \mathrm{O}_{3}+\mathrm{CO}_{2}
$$

Cerium oxide as an oxidation catalyst also lowers the carbon combustion activation temperature and thus enhances hydrocarbon oxidation, promoting complete combustion. The variation of $\mathrm{HC}$ emissions with load for diesel, diesel with $35 \mathrm{ppm}$ of cerium oxide nanoparticle, and that with cerium oxide nanoparticles and DDSA was also measured and is illustrated in Figure 11. Figure 11 shows that the addition of surfactant DDSA along with the nanoparticles leads to a further reduction in the $\mathrm{HC}$ emissions.

3.3.2. $\mathrm{NO}_{x}$ Emissions. The $\mathrm{NO}_{x}$ emissions were found to be decreased by a maximum of $30 \%$, on the addition of cerium oxide nanoparticles in diesel, especially at higher load, as shown in Figure 12. It was observed that the reduction in the $\mathrm{NO}_{x}$ emissions increases with the increase in the concentration of ceria from $5 \mathrm{ppm}$ to $35 \mathrm{ppm}$. Cerous oxide $\left(\mathrm{Ce}_{2} \mathrm{O}_{3}\right)$ formed after the oxidation of hydrocarbon and soot gets reoxidized to $\mathrm{CeO}_{2}$ through the reduction of nitrogen oxide as per the following reaction [28]:

$$
\mathrm{Ce}_{2} \mathrm{O}_{3}+\mathrm{NO} \longrightarrow 2 \mathrm{CeO}_{2}+\frac{1}{2} \mathrm{~N}_{2} \text {. }
$$

Figure 13 shows the comparison of $\mathrm{NO}_{x}$ emissions for diesel, with that of nanoparticle added diesel and DDSA treated nanoparticle added diesel. Nitrogen oxide emission was found to be decreased with the addition of cerium oxide nanoparticle and further reduction up to $50 \%$ with the addition of $5 \%$ volume fraction of DDSA treated nanoparticle. With the addition of DDSA the viscosity of the fuel decreases and better dispersion of nanofuel is obtained, leading to further reduction in the emissions.

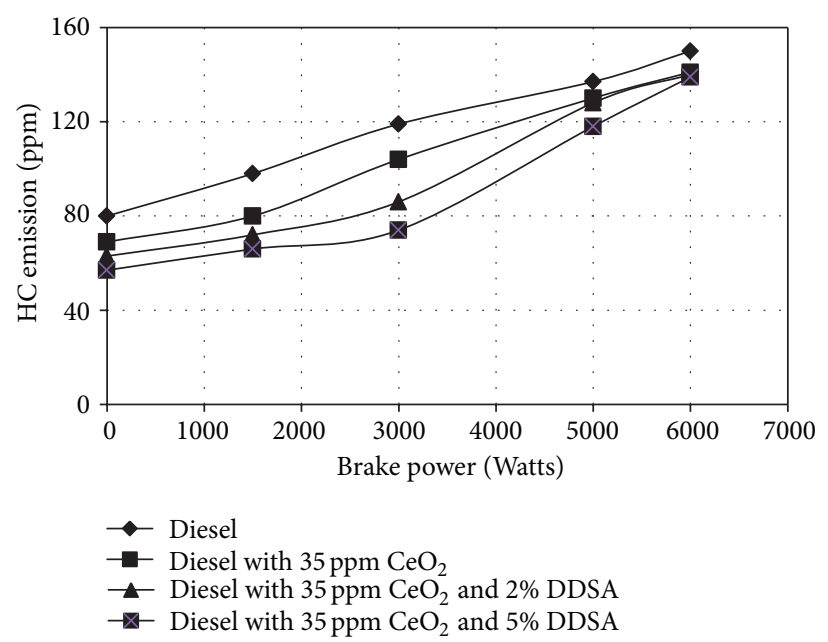

FIGURE 11: Variation of HC emissions with load for diesel and modified diesel ( $35 \mathrm{ppm}$ of $\mathrm{CeO}_{2}$ ) for different vol \% of surfactant (DDSA).

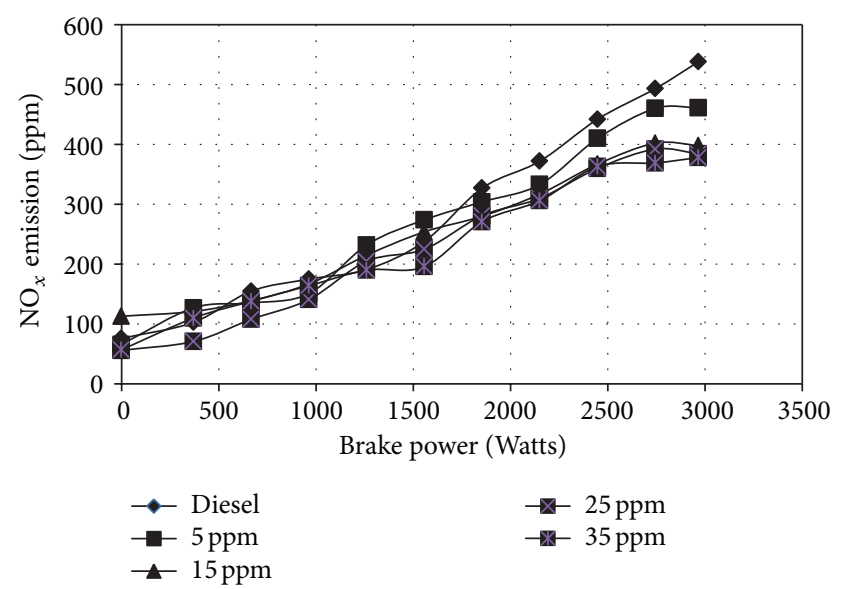

FIGURE 12: Variation of $\mathrm{NO}_{x}$ emissions with load for diesel and modified diesel.

\section{Conclusions}

The present work aims at the synthesis of the cerium oxide nanoparticles and investigation on the effect of nanoparticles on various physicochemical properties of diesel and engine performance and emissions. Characterization techniques such as EDS, XRD, and TEM have been used for studying the properties of the cerium oxide nanoparticle prepared by the precipitation method. Surfactant has been added to the diesel for improving the stability of the nanofluid, used for the experimentation. Load test has been done in the diesel engine to investigate the effect of catalytic nanoparticles on the efficiency and the emissions from the engine. Based on the experiments the following conclusions were drawn.

(1) The flash point and fire point increase with the dosing level of cerium oxide nanoparticles in diesel; 


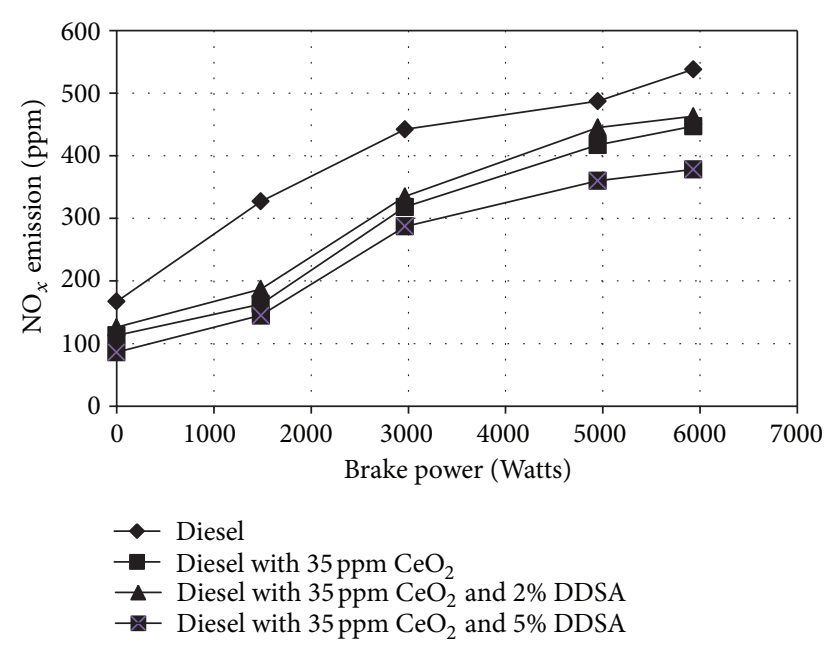

FIgURE 13: Variation of $\mathrm{NO}_{x}$ emissions with load for diesel and modified diesel (35 ppm of $\mathrm{CeO}_{2}$ ) for different vol \% of surfactant (DDSA).

(2) even though kinematic viscosity increases with the catalytic nanoparticle addition in fuel, it was found to be decreasing with the addition of surfactant DDSA;

(3) the results of the load test on the diesel show that the efficiency is increased up to $5 \%$ and a reduction of $\mathrm{HC}$ and $\mathrm{NO}_{x}$ emissions by $45 \%$ and $30 \%$, respectively, especially at higher load;

(4) the reduction in the emissions is proportional to the dosing level of nanoparticles in the diesel and optimum dosing level of $35 \mathrm{ppm}$ of catalytic nanoparticles was observed.

\section{Conflict of Interests}

The authors declare that there is no conflict of interests.

\section{Acknowledgments}

The authors are very much acknowledged to Dr. C. B. Sobhan and Dr. N. Sandhyarani of School of Nano Science and Technology, NIT Calicut for their extreme help and directions throughout the work. They are also thankful to Mrs. Parvathy R. Chandran and Ms. Manjusha Mathew for adorning his work by their pleasant advices and motivations.

\section{References}

[1] K. J. Baumgard and D. B. Kittelson, "The Influence of a Ceramic Particle Trap on the Size Distribution of Diesel Particles," SAE Technical Paper 850009, 1985.

[2] G. Lepperhoff and G. Kroon, "Impact of Particulate Traps on the Hydrocarbon Fraction of Diesel Particles," SAE Technical Paper 850013, 1985.

[3] M. Gürü, U. Karakaya, D. Altiparmak, and A. Alicilar, "Improvement of Diesel fuel properties by using additives," Energy Conversion and Management, vol. 43, no. 8, pp. 1021$1025,2002$.
[4] H. N. Kim and B. C. Choi, "Effect of ethanol-diesel blend fuels on emission and particle size distribution in a common-rail direct injection diesel engine with warm-up catalytic converter," Renewable Energy, vol. 33, no. 10, pp. 2222-2228, 2008.

[5] E. W. de Menezes, R. da Silva, R. Cataluña, and R. J. C. Ortega, "Effect of ethers and ether/ethanol additives on the physicochemical properties of diesel fuel and on engine tests," Fuel, vol. 85, no. 5-6, pp. 815-822, 2006.

[6] L. Xiaolu, C. Hongyan, Z. Zhiyong, and H. Zhen, "Study of combustion and emission characteristics of a diesel engine operated with dimethyl carbonate," Energy Conversion and Management, vol. 47, no. 11-12, pp. 1438-1448, 2006.

[7] X. Shi, Y. Yu, H. He, S. Shuai, J. Wang, and R. Li, "Emission characteristics using methyl soyate-ethanol-diesel fuel blends on a diesel engine," Fuel, vol. 84, no. 12-13, pp. 1543-1549, 2005.

[8] P. Satgé de Caro, Z. Mouloungui, G. Vaitilingom, and J. C. Berge, "Interest of combining an additive with diesel-ethanol blends for use in diesel engines," Fuel, vol. 80, no. 4, pp. 565-574, 2001.

[9] H. R. Chao, T. C. Lin, M. R. Chao, F. H. Chang, C. I. Huang, and C. B. Chen, "Effect of methanol-containing additive on the emission of carbonyl compounds from a heavy-duty diesel engine," Journal of Hazardous Materials, vol. 73, no. 1, pp. 3954,2000

[10] P. Dutta, S. Pal, M. S. Seehra, Y. Shi, E. M. Eyring, and R. D. Ernst, "Concentration of $\mathrm{Ce}^{3+}$ and oxygen vacancies in cerium oxide nanoparticles," Chemistry of Materials, vol. 18, no. 21, pp. 5144-5146, 2006.

[11] V. Sajith, M. Sandhya, and C. B. Sobhan, "An investigation into the effect of inclusion of cerium oxide nanoparticles on the physicochemical properties of diesel oil," in Proceedings of the ASME International Mechanical Engineering Congress and Exposition (ASME IMECE '06), pp. 333-338, Materials Division Publication, 2006.

[12] V. Sajith, C. B. Sobhan, and G. P. Peterson, "Experimental investigations on the effects of cerium oxide nanoparticle fuel additives on biodiesel," Advances in Mechanical Engineering, vol. 2010, Article ID 581407, 6 pages, 2010.

[13] V. A. M. Selvan, R. B. Anand, and M. Udayakumar, "Effects of cerium oxide nanoparticle addition in diesel and dieselbiodiesel-ethanol blends on the performance and emission characteristics of a CI engine," Journal of Engineering and Applied Sciences, vol. 4, no. 7, pp. 1819-6608, 2009.

[14] H. I. Chen and H. Y. Chang, "Homogeneous precipitation of cerium dioxide nanoparticles in alcohol/water mixed solvents," Colloids and Surfaces A, vol. 242, no. 1-3, pp. 61-69, 2004.

[15] H. I. Chen and H. Y. Chang, "Synthesis of nanocrystalline cerium oxide particles by the precipitation method," Ceramics International, vol. 31, no. 6, pp. 795-802, 2005.

[16] V. K. Ivanov, O. S. Polezhaeva, G. P. Kopitsa, A. E. Baranchikov, and Y. D. Tret'yakov, "Fractal structure of ceria Nanoparticles," Inorganic Materials, vol. 44, no. 3, pp. 272-277, 2008.

[17] H. Gu and M. D. Soucek, "Preparation and characterization of monodisperse cerium oxide nanoparticles in hydrocarbon solvents," Chemistry of Materials, vol. 19, no. 5, pp. 1103-1110, 2007.

[18] T. Masui, K. Fujiwara, K. I. Machida, G. Y. Adachi, T. Sakata, and H. Mori, "Characterization of cerium(IV) oxide ultrafine particles prepared using reversed micelles," Chemistry of Materials, vol. 9, no. 10, pp. 2197-2204, 1997.

[19] S. T. Aruna and A. S. Mukasyan, "Combustion synthesis and nanomaterials," Current Opinion in Solid State and Materials Science, vol. 12, no. 3-4, pp. 44-50, 2008. 
[20] L. Mädler, W. J. Stark, and S. E. Pratsinis, "Flame-made ceria nanoparticles," Journal of Materials Research, vol. 17, no. 6, pp. 1356-1362, 2008.

[21] G. Skillas, Z. Qian, U. Baltensperger, U. Matter, and H. Burtscher, "Influence of additives on the size distribution and composition of particles produced by diesel engines," Combustion Science and Technology, vol. 154, no. 1, pp. 259-273, 2000.

[22] J. Lahaye, S. Boehm, P. Chambrion, P. Chambrion, and P. Ehrburger, "Influence of cerium oxide on the formation and oxidation of soot," Combustion and Flame, vol. 104, no. 1-2, pp. 199-207, 1996.

[23] J. P. A. Neeft, M. Makkee, and J. A. Moulijn, "Diesel particulate emission control," Fuel Processing Technology, vol. 47, no. 1, pp. 1-69, 1996.

[24] B. Stanmore, J.-F. Brilhac, and P. Gilot, "The Ignition and Combustion of Cerium Doped Diesel Soot," SAE Technical Paper 1999-01-0115, 1999.

[25] H. Jung, D. B. Kittelson, and M. R. Zachariah, “The influence of a cerium additive on ultrafine diesel particle emissions and kinetics of oxidation," Combustion and Flame, vol. 142, no. 3, pp. 276-288, 2005.

[26] ASTM D-445, "Test method for kinematic viscosity of transparent and opaque liquids".

[27] ASTM D92-05a, "Test method for flash and fire points by Cleveland open cup tester".

[28] G. R. Rao and B. G. Mishra, "Structural, redox and catalytic chemistry of Ceria based materials," Bulletin of the Catalysis Society of India, vol. 2, pp. 122-134, 2003. 

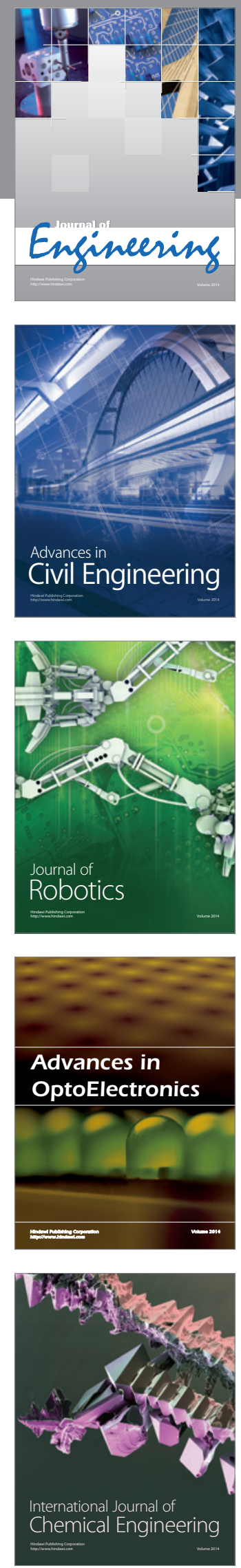

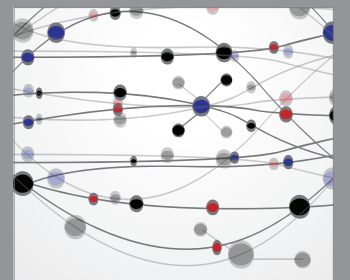

The Scientific World Journal
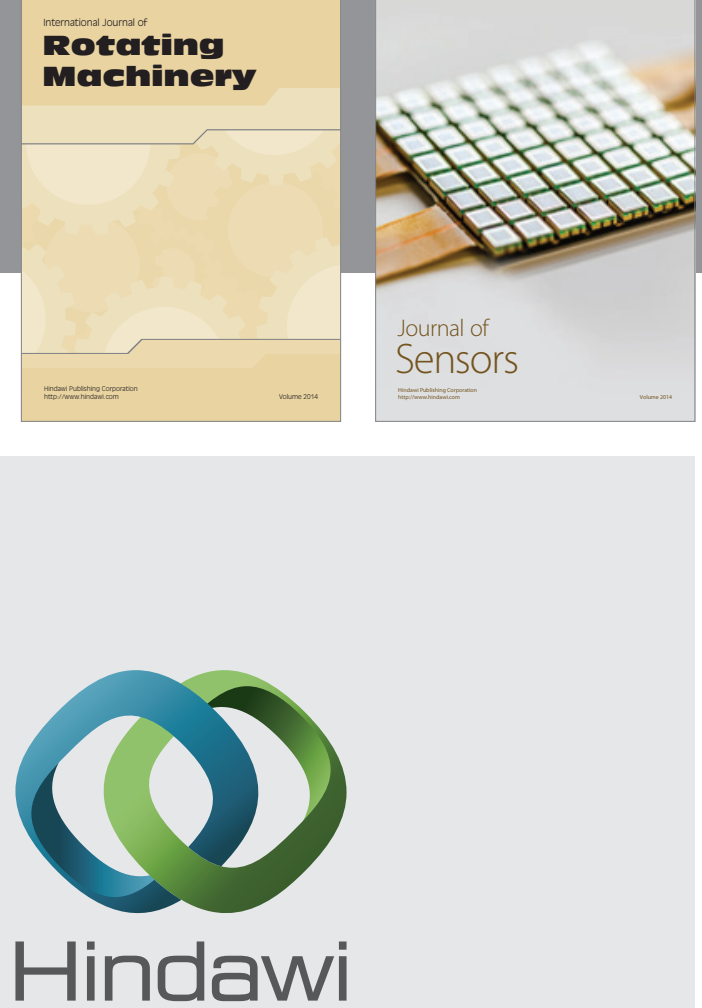

Submit your manuscripts at http://www.hindawi.com
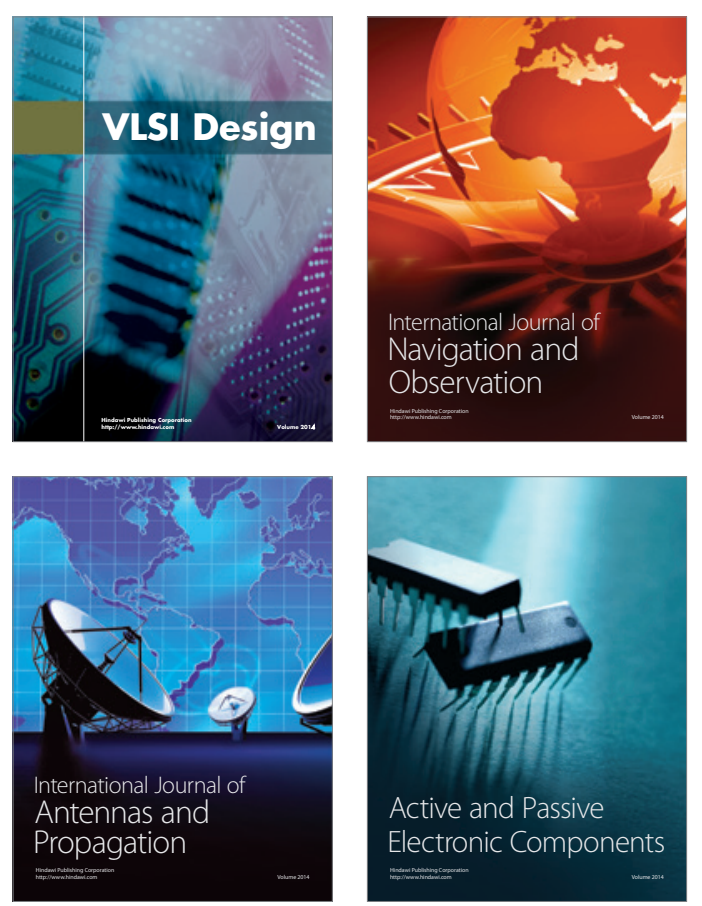
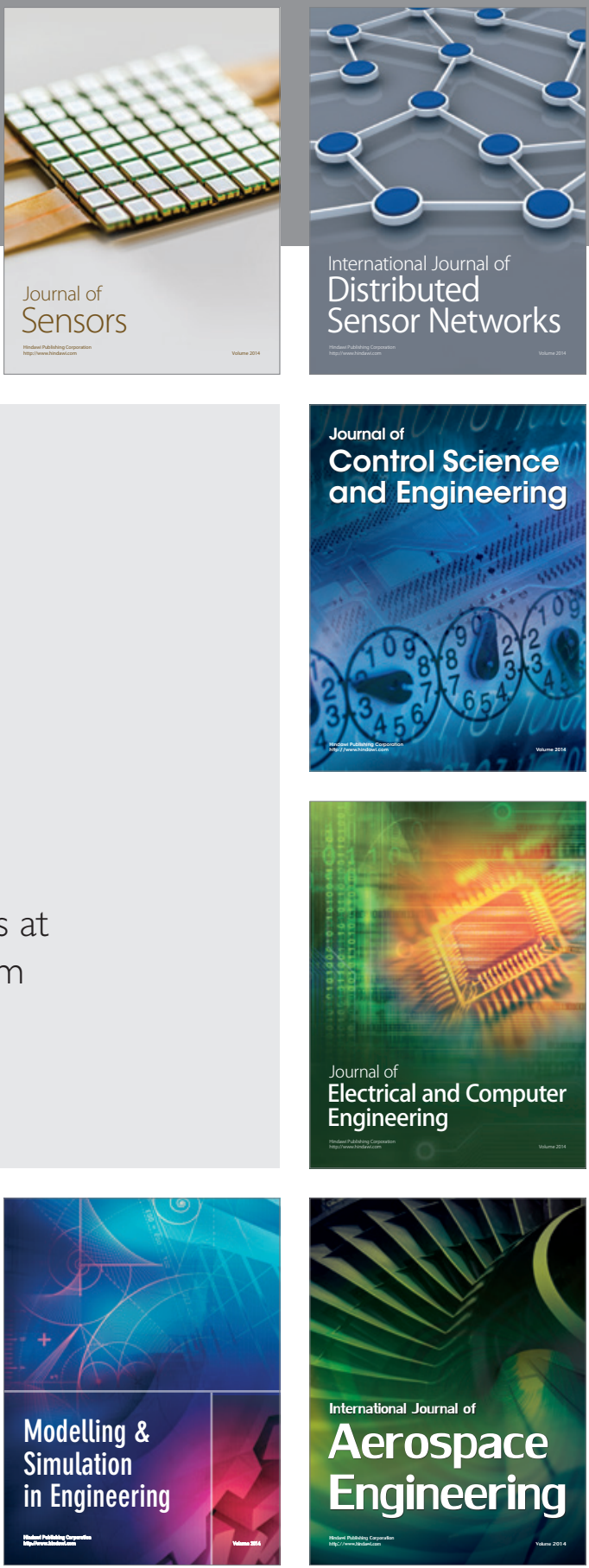

Journal of

Control Science

and Engineering
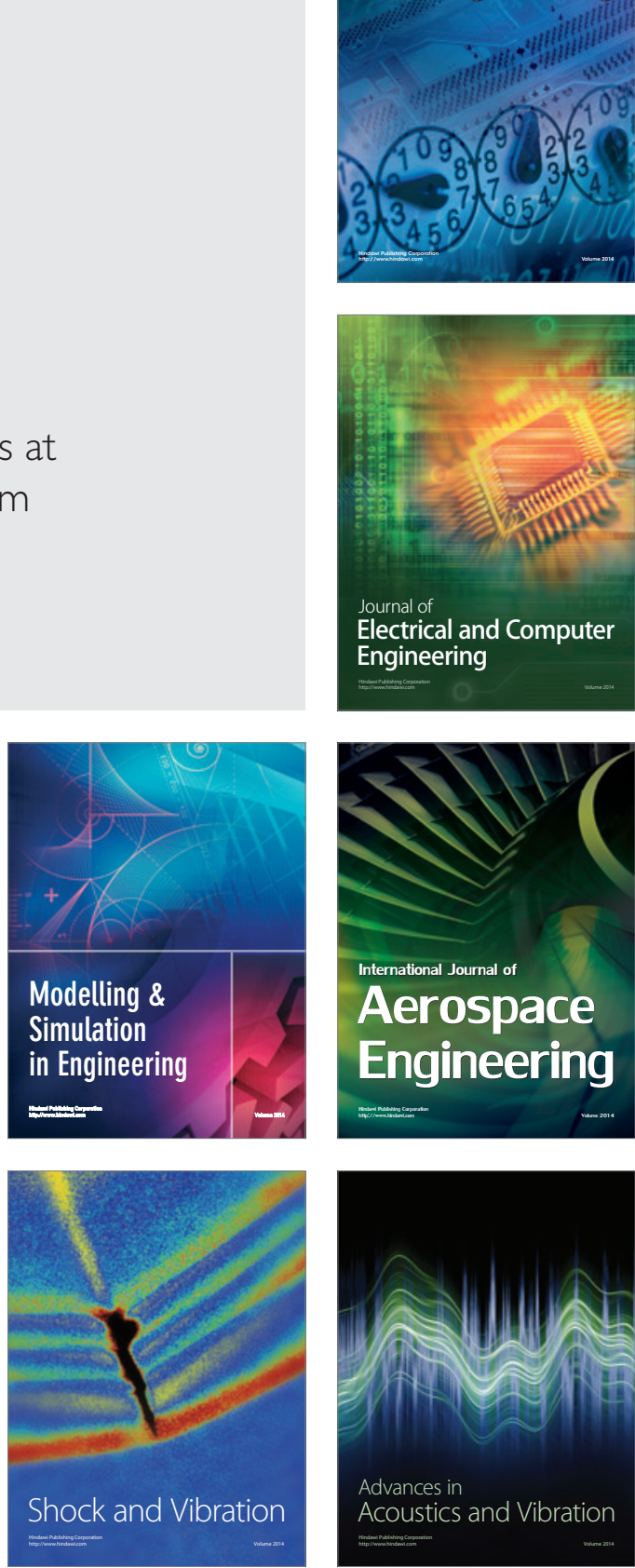